\title{
Effect of the Jianpi Bushen Prescription on the expression of SHP-1, Wnt3a, and AP-1 proteins in chemically damaged mice
}

\author{
D.C. He*, J.J. Xiao*, Y. Zhang, H. Lin, X.J. Ding and Y. Tu \\ Wuhan General Hospital of Guangzhou Military Region, \\ Wuhan, Hubei, China \\ *These authors contributed equally to this study. \\ Corresponding author: D.C. He \\ E-mail: xiaojingjing5961@126.com
}

Genet. Mol. Res. 13 (2): 3201-3208 (2014)

Received May 22, 2013

Accepted September 20, 2013

Published April 25, 2014

DOI http://dx.doi.org/10.4238/2014.April.25.5

\begin{abstract}
This study investigated the effect of the Jianpi Bushen Prescription (JBP) on the expression of 3 major proteins in chemically damaged model mice. The 3 proteins were the Wnt3a, the SHP-1, and the transcription factors (NF-E2, c-jun, and c-fos) of the AP-1 protein family. Kunming mice were randomly divided into chemically damaged group $(\mathrm{N}=48)$, which received an abdominal injection of $(100 \mathrm{mg} / \mathrm{kg})$ cyclophosphamide once a day for 3 consecutive days, and control group $(\mathrm{N}=12)$, which received the same amount of saline. Then, the chemically damaged mice were randomly divided into chemically damaged model group $(\mathrm{N}=12)$, which received $0.2 \mathrm{~mL} / 10 \mathrm{~g}$ of saline twice a day for 9 days, positive control group $(\mathrm{N}=12)$, which received $0.2 \mathrm{~mL} / 10 \mathrm{~g}$ of the e-jiao slurry (EJS) compound twice a day for 9 days, low dose JBP group $(\mathrm{N}=12)$, which received $0.1 \mathrm{~g} / \mathrm{kg}$ suspension JBP $(100 \%$ concentration) twice a day for 9 days and high dose JBP group $(\mathrm{N}=12)$, which received $0.1 \mathrm{~g} / \mathrm{kg}$ suspension JBP (200\% concentration) twice a day for 9 days. The bilateral femur and tibia bone marrow were collected from the mice in all groups. The protein expression of the specified
\end{abstract}


proteins and transcription factors in the bone marrow mononuclear cells were detected by Western blot analysis. The results showed that the protein expression of Wnt3a was significantly downregulated in the chemically damaged model group compared to the control group $(\mathrm{P}<$ 0.05). The low dose JBP, high dose JBP, and e-jiao slurry treatments significantly upregulated the protein expression of Wnt3a compared to the chemically damaged model group $(\mathrm{P}<0.05)$, with the low dose JBP producing the best results. Compared to the control group, the protein expressions of SHP-1, c-fos, c-jun, and NF-E2 were significantly higher in the chemically damaged model group (all $\mathrm{P}<0.05$ ). The protein expressions of SHP-1, c-fos, c-jun, and NF-E2 were significantly lower in the chemically damaged model + the low dose JBP, chemically damaged model + high dose JBP, or chemically damaged model + EJS group compared to chemically damaged model (all $\mathrm{P}<0.05$ ), with the low dose JBP producing the best results. These results indicate that JBP regulates the expressions of SHP-1, Wnt3a, and AP-1 proteins in chemically damaged mice.

Key words: Jianpi Bushen Prescription; Chemical damage; SHP-1 protein; Wnt3a protein; Transcription regulatory protein

\section{INTRODUCTION}

With scientific and technological developments, humans are exposed to increasing amounts of chemicals in everyday life and industry, with many of these chemicals having potential carcinogenicity. The hematopoietic and immune systems of the body are where damage from carcinogens primarily occurs (Kuhn, 2002; Kemp et al., 2011). For instance recent publications suggest that during the Gulf War (in Iraq), there was a 3-fold increase in childhood leukemia, a 4-fold increase in cancer, a 9-fold increase in cancer mortality, and a 3 -fold increase reproductive abnormalities in the region because of chemical and radiation damage.

Chemical damage suppresses bone marrow growth, particularly cytopenia and immune function; however certain cell growth factors may be used to counteract these phenomena (Puhalla et al., 2012). However, the use of cell growth factors has some limitations. For instance, this treatment is expensive and may only be used for agranulocytosis, as it has no effect on thrombocytopenia. Furthermore, and the latest research shows that cell growth factors may inhibit cytotoxic agents that induce apoptosis, leading to the continued survival of tumor cells and counteraction of chemical drugs. Therefore, it is extremely important to identify safe, efficient, and low toxicity drugs that are able to promote the growth of hematopoietic stem cells.

Jianpi Bushen Prescription (JBP, previously known as Digan Oral Liquid) has a protective function against radiation and chemical damage. Early clinical trials have indicated that this prescription may improve peripheral blood levels and enhance immunological functioning. Existing published animal experiments have demonstrated the protection mechanism of JBP on radiation damage. For instance, it operates as a marrow colony stimulating factor, 
promotes the expressions of apoptosis-related genes in the marrow and spleen, and causes splenic histopathological changes. In recent years, experimental studies have focused on the protective effects of JBP against chemical damage (Fossett, 2013); however, the relationship between chemical damage and the cell signaling pathways remain unclear. In part, this is due to the lack of systematic and objective experiments in research focused on traditional Chinese medicines. This study selected a series of related cell signaling pathway indicators, and systematically evaluated the protective effect of JBP on chemical damage to elucidate its protective mechanism and to provide statistically sound experimental data supporting its clinical application.

\section{MATERIAL AND METHODS}

\section{Study animals}

Sixty healthy male and female Kunming mice were selected and weighed 18 to 22 g. These mice were provided by the Hubei Province Experimental Animal Research Center.

\section{Drug preparation}

JBP was prepared from a large dose of prepared rehmannia root and honey-fried licorice root, supplemented with angelica, radix astragalus, and tangerine peel. The prescription was prepared by the Department of Pharmaceutical Preparation in the Wuhan General Hospital of Guangzhou Military Region hospital. The extract liquid of prescription was subject to water decoction and filtration. Then, the extract liquid of prescription was concentrated to $100 \%$ (every milliliter contained $1 \mathrm{~g}$ of the crude drug) and 200\% (every milliliter contained 2 g crude drug) liquid medicine. It was then packaged and stored. The e-jiao slurry (EJS) compound was provided by Shandong DEEJ Co., Ltd. (batch No. 20101008).

\section{Main reagents and apparatus}

The erythrocyte splitting liquor was purchased from BA Phil Wuhan Company (China). A DYCZ-40 Electrorotation Generator was used, which was manufactured by the Beijing LiuYi Company, China. Trizol was purchased from Invitrogen Company (American). Reverse transcriptase and the kit to extract first chain synthetic cDNA was purchased from Fermentas Company (USA). Polyvinylidene fluoride (PVDF) Film was purchased from Millipore Acrylamide (American) and PMSF was purchased from Amresco (American). ECL substrate solution was purchased from Thermo NC15079 (American). Other chemical reagents were obtained from traditional Chinese medicine sources. The micropipette was purchased from the Eppendorf Company (Germany). The electro-thermostatic blast oven was purchased from Shanghai Jing Hong Laboratory Instrument Co., Ltd. (China). The PCR instrument was purchased from Dongsheng Innovation Biotechnology Ltd. Co. (China). The spectrophotometer was purchased from ShangHai Sunny HenPing Scientific Instrument Co., Ltd. (China). The real-time fluorescent quantitative PCR was purchased from Imullina Eco (China). The horizontal gel electrophoresis apparatus and spectrophotometer were purchased from Bejing Junyi-Dongfang Electrophoresis Equipment Co., Ltd. (China). 


\section{Models and groups}

Sixty Kunming mice were fed ad libitum for 1 week. Twelve mice were randomly selected as the normal (control) group. All other mice were given an abdominal injection of cyclophosphamide $(100 \mathrm{mg} / \mathrm{kg})$ once a day for 3 consecutive days to induce the chemical damage. The standard for chemical damage in mice was that the appetite and activity of mice were significantly decreased and the white blood cell count was significantly reduced compared with control group (normal control mice: $12.61 \times 10^{9} / \mathrm{L}$; chemically damaged mice: 7.41 x $10^{9} / \mathrm{L}$ ). The success rate of the chemical damage model was $100 \%$. Then, the chemically damaged mice were randomly divided into chemically damaged model group $(\mathrm{N}=12)$, which received $0.2 \mathrm{~mL} / 10 \mathrm{~g}$ saline twice a day for 9 days, positive control group $(\mathrm{N}=12)$, which received $0.2 \mathrm{~mL} / 10 \mathrm{~g}$ EJS compound twice a day for 9 days, low dose JBP group $(\mathrm{N}=12)$, which received $0.1 \mathrm{~g} / \mathrm{kg}$ suspension JBP $(100 \%$ concentration), and high dose JBP group (N $=12$ ), which received $0.1 \mathrm{~g} / \mathrm{kg}$ suspension JBP (200\% concentration) twice a day for 9 days. On the 9th day, the mice were sacrificed to collect the bilateral femur and tibia bone marrow.

\section{Western blot analysis}

Western blot analysis was used to detect the expressions of the SHP-1 protein, the Wnt3a protein, and the transcription factors (NF-E2, c-jun, and c-fos) of the AP-1 protein family. The tissue weight of the bilateral femur and tibia bone marrow was calculated after collecting the samples from the specimens. Then, the radio-immunoprecipitation assay erythrocyte splitting liquor was added to the cell suspension and the mixture was blended. The mixture was centrifuged at $4^{\circ} \mathrm{C}$ for $5 \mathrm{~min}$ to remove the supernatant. The supernatant was then stored at $-20^{\circ} \mathrm{C}$ until use.

The protein concentrations were then determined. The same quantity of erythrocyte splitting liquor (volume $\mathrm{x}$ protein concentration) was used, and the same volume of $2 \mathrm{X}$ electrophoresis loading buffer was also added. After 3 min of boiling the mixture in water, protein samples were loaded onto the separation gel, and subjected to electrophoresis. The mixture was placed on a transmembrane, and then immersed in a PVDF membrane in TBST (i.e., sealing fluid), that contained $5 \%$ of BSA. Then the mixture was shaken at room temperature for $2 \mathrm{~h}$. Use sealing fluid was diluted to the levels of the corresponding primary antibodies, and incubated overnight at $4^{\circ} \mathrm{C}$. Secondary antibodies, which were marked with HRP-labels, were incubated, and then shaken at room temperature for $2 \mathrm{~h}$. ECL substrate fluid was added, and then the fluorescence band was observed. This was captured by x-ray film squash, development, and fixation. The film was scanned, and the Bandscan software analysis was used, with gene expression being represented by the gray value of the gene (GAPDH).

\section{Statistical analysis}

SPSS 11.0 was used for statistical data processing. All data are reported as means \pm SD. Differences among groups were examined for statistical significance by the F-test and single factor analysis of variance between multiple groups, while the $t$-test and least significant difference analysis of variance were used between any 2 groups. A P value of less than 0.05 denotes the presence of a statistically significant difference. 


\section{RESULTS}

\section{Effect of JBP on the protein expression of Wnt3a, SHP-1, c-fos, c-jun, and NF-E2 in chemically damaged mice}

As shown in Figure 1, compared to the control group, the protein expression of Wnt3a was significantly downregulated in the chemically damaged model group $(\mathrm{P}<0.05)$. The low dose JBP, high dose JBP, and EJS treatments significantly upregulated the protein expression of Wnt3a compared to the chemically damaged model group given sodium chloride $(\mathrm{P}<$ 0.05). The low dose JBP produced the best results. Compared to the control group, the protein expressions of SHP-1, c-fos, c-jun, and NF-E2 were significantly higher in the chemically damaged model group (all $\mathrm{P}<0.05$ ). The protein expressions of SHP-1, c-fos, c-Jun, and NFE2 were significantly lower in the chemically damaged model + low dose JBP, chemically damaged model + high dose JBP, or chemically damaged model + EJS treatment groups (all P $<0.05)$. The low dose JBP produced the best results.
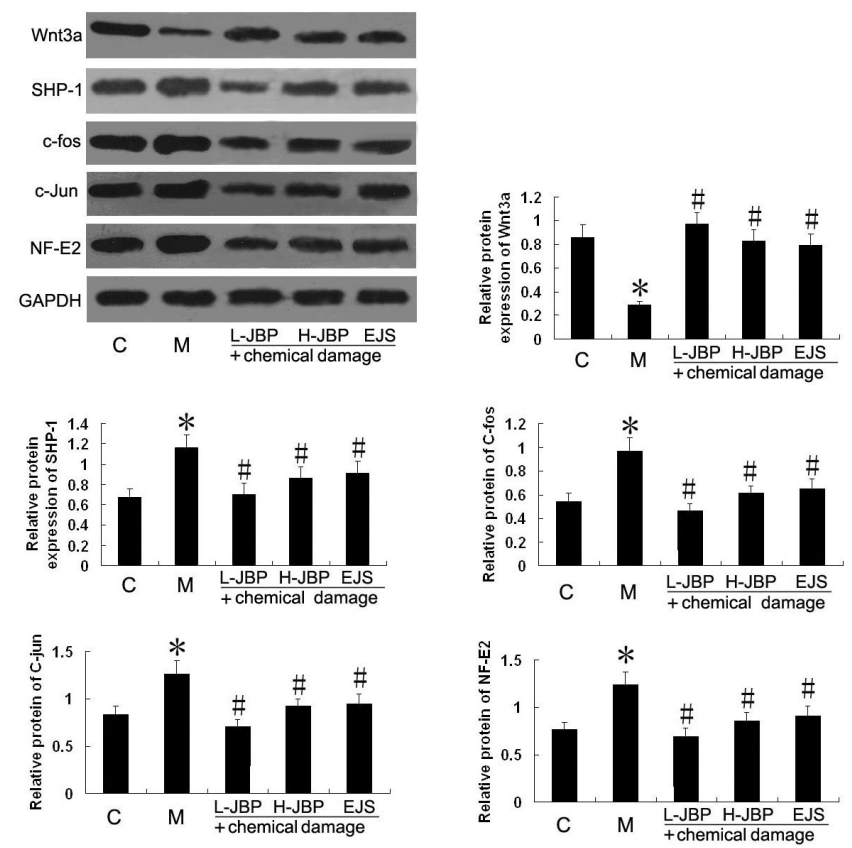

Figure 1. Effect of the traditional Chinese drug Jianpi Bushen Prescription (JBP) on the protein expression of Wnt3a, SHP-1, c-fos, c-jun, and NF-E2 in chemically damaged mice. Kunming mice were randomly divided into chemically damaged group, which received an abdominal injection of $100 \mathrm{mg} / \mathrm{kg}$ cyclophosphamide once a day for 3 days and control group, which received the same amount of saline. Then, the chemically damaged mice were randomly divided into chemically damaged model group, which received $0.2 \mathrm{~mL} / 10 \mathrm{~g}$ saline twice a day for 9 days, positive control group, which received $0.2 \mathrm{~mL} / 10 \mathrm{~g}$ e-jiao slurry (EJS) compound twice a day for 9 days, low dose JBP group, which received $0.1 \mathrm{~g} / \mathrm{kg}$ suspension JBP $(100 \%$ concentration) twice a day for 9 days, and high dose JBP group, which received $0.1 \mathrm{~g} / \mathrm{kg}$ suspension JBP $(200 \%$ concentration) twice a day for 9 days. The bilateral femur and tibia bone marrow were collected and the protein expressions of Wnt3a, SHP-1, c-fos, c-jun, and NF-E2 in the cells were tested by Western blot analysis. $\mathrm{C}=$ control group; $\mathrm{M}=$ chemically damaged model group; low JBP = low dose of JBP $(100 \%)$ group; high JBP $=$ high dose of JBP $(200 \%)$ group; EJS = EJS compound group. Data are reported as means \pm $\mathrm{SD}(\mathrm{N}=12)$. ${ }^{*} \mathrm{P}<0.05$, compared to control group; ${ }^{*} \mathrm{P}<0.05$, compared to chemically damaged model group. 


\section{DISCUSSION}

Traditional Chinese medicine states that chemically damage is the "deficiency of spleen and kidney, lack of blood and gas". The principle of treatments used to address chemically damage is to "benefit gas and enrich blood". Traditional Chinese medicine considers the spleen to be the source of gas and blood biochemistry, while the kidney regulates the bones, fresh marrow, and stores essential substances. Therefore, it is important to fortify the spleen and supplement the kidney.

JBP is an empirical formula established by the Department of Integrated Chinese and Western Medicine of Wuhan General Hospital of Guangzhou Military Region of China, and is mainly composed of large dose of prepared rehmannia root and honey-fried licorice root, supplemented with angelica, radix astragalus, and tangerine peel. The various drugs are compatible towards, fortifying the spleen and supplementing the kidney, and so nourish the blood and marrow. Modern pharmacology studies have confirmed that rehmannia glutinosa is able to recover the hematopoietic function of bone marrow after suppression radiation damage. This compound is able to both promote the proliferation of hemopoietic stem cells and improve immune function (Ma et al., 2010). Angelica polysaccharide (AP) plays a significant role toward promoting the proliferation and differentiation of liver hematopoietic cells and myeloid hematopoietic progenitor cells. This compound is able to counteract conditions when peripheral blood cell numbers decline and bone marrow is suppressed. It also improves the hematopoietic microenvironment to regulate hematopoietic function (Liu et al., 2010; Hou et al., 2012). Radix astragalus has a comprehensive and sustained effect in elevating whole blood cells, recovering red blood cell, while blood cell, and platelet levels to normal. In addition, it promotes the secretion of hematopoietic cytokine, stimulating the function of the hematopoietic system, and counteracting bone marrow suppression caused by chemotherapy and radiotherapy (Gao et al., 2006; Zheng et al., 2011). In summary, modern medicine has demonstrated that the spleen and kidney have the dual role of promoting the regulation of hematopoietic cell proliferation and immune function.

Signaling molecules enhance the capacity of hematopoietic stem cell self-renewal through different signaling pathways, and have become the focus of scientific research in recent years. Previous studies have shown that blockage of the Wnt, JAK signaling pathway and the excessive expression of transcription factors play a vital role in the self-renewal of hematopoietic cells. The Wnt signaling pathway plays an important role in the multifaceted development process of biological structure formation, cell differentiation, and the morphogenesis and evolution of tumors (Staal and Luis, 2010; Ichii et al., 2012). Wnt/ $\beta$-catenin is one of the classical Wnt signaling pathways involved in cell growth and development. Disorders of Wnt/ $\beta$-catenin cause abnormal cell growth and prosoplasia (Ming et al., 2012). Wnt3a is an important promoter gene in the $\mathrm{Wnt} / \beta$-catenin signaling pathway. It maintains the self-renewal capacity of hematopoietic cells and amplifies their numbers. This is achieved by activating the Wnt signaling pathway and increasing the expression of $\beta$-catenin, so as to enhance the self-renewal capacity of hematopoietic stem cells (Kawaguchi-Ihara et al., 2008; Zhang et al., 2012). The JAK/STAT signaling pathway plays a major role in facilitating hematopoietic cell growth, proliferation, differentiation, and apoptosis (Hankey, 2009; Kiu and Nicholson, 2012). Hematopoietic growth factors combine with the corresponding receptor through the JAK/ STAT pathway, to adjust intracellular signal transduction; this action affects both DNA expression and hematopoietic cell growth and function (Baker et al., 2007). Previous studies have 
found that SHP-1 phosphorylation inhibits the JAK/STAT signaling pathway by negatively regulating JAK kinase activity. In comparison, the downregulation of SHP-1 protein expression might weaken its inhibitory effect on the JAK/STAT signaling pathway; thus, promoted the proliferation and differentiation of hematopoietic cells (López-Ruiz et al., 2011; Chen et al., 2012). The intracellular signaling pathway acts as a bridge between the cell surface and the special intranuclear gene, playing a decisive role on the response of excitable cells to external stimulation. The transcriptional regulation of genes forms one aspect of intracellular signaling pathways. NF-E2, c-jun, and c-fos transcription factor proteins combine with leucine to form heterodimers or homodimers, to form the transcriptional regulatory protein AP-1. AP-1 plays a key role in tumor transformation, which is reversed when it binds to DNA. AP-1 regulates the expression of downstream target genes at the transcriptional level, and is involved in cell proliferation and differentiation (Turpaev, 2006; Lee et al., 2012). Studies have shown that high NF-E2, c-jun, and c-fos expression is closely linked with the occurrence of a variety of cancers (Guinea-Viniegra et al., 2012). By weakening the transcription of the NF-E2, c-jun, and c-fos protein, and by reducing the synthesis of AP-1, it might be possible to regulate the expressions of the proliferation gene and differentiation gene of hematopoietic cells, and hence promote the generation of blood cells.

This study demonstrated that intervention with JBP promotes Wnt3a protein expression and weakens the expressions of SHP-1 protein and the transcription of NF-E2, c-jun, and c-fos factors in the AP-1 protein family. This study indicated that JBP regulates the expression of proliferation and differentiation of hematopoietic cells by activating the Wnt and JAK signaling pathways and by restraining the excessive expression of transcription factors; thus, JBP promotes the formation of blood cells. This study also found that low-dose JBP group was the most effective at counteracting chemical damage compared to the high-dose group. It appeared that high concentrations of the drug weakened the transduction and transcription of the signal pathway, which might have been caused by the large quantities of rehmannia and licorice in the JBP preventing stomach transportation and absorption. In conclusion, JBP has a certain dose-effect relationship with the treatment of bone marrow suppression caused by chemical damage, rather than the large dose dependency.

\section{ACKNOWLEDGMENTS}

Research supported by the Military Medicine Special Task (\#10zyz218) and the National Natural Science Foundation of China (\#81273905).

\section{REFERENCES}

Baker SJ, Rane SG and Reddy EP (2007). Hematopoietic cytokine receptor signaling. Oncogene 26: 6724-6737.

Chen KF, Tai WT, Hsu CY, Huang JW, et al. (2012). Blockade of STAT3 activation by sorafenib derivatives through enhancing SHP-1 phosphatase activity. Eur. J. Med. Chem. 55: 220-227.

Fossett N (2013). Signal transduction pathways, intrinsic regulators, and the control of cell fate choice. Biochim. Biophys. Acta 1830: 2375-2384

Gao QT, Cheung JK, Li J, Chu GK, et al. (2006). A Chinese herbal decoction, Danggui Buxue Tang, prepared from Radix Astragali and Radix Angelicae Sinensis stimulates the immune responses. Planta Med. 72: 1227-1231.

Guinea-Viniegra J, Zenz R, Scheuch H, Jiménez M, et al. (2012). Differentiation-induced skin cancer suppression by FOS, p53, and TACE/ADAM17. J. Clin. Invest. 122: 2898-2910.

Hankey PA (2009). Regulation of hematopoietic cell development and function by Stat3. Front Biosci. 14: 52735290.

Genetics and Molecular Research 13 (2): 3201-3208 (2014)

CFUNPEC-RP www.funpecrp.com.br 
Hou H, Bao Y, Li Q and Shi W (2012). Preparation of blood-deficient model and research of angelica polysaccharide on enriching blood in chickens. Evid. Based. Complement. Alternat. Med. 2012: 965947.

Ichii M, Frank MB, Iozzo RV and Kincade PW (2012). The canonical Wnt pathway shapes niches supportive of hematopoietic stem/progenitor cells. Blood 119: 1683-1692.

Kawaguchi-Ihara N, Murohashi I, Nara N and Tohda S (2008). Promotion of the self-renewal capacity of human acute leukemia cells by Wnt3A. Anticancer Res. 28: 2701-2704.

Kemp K, Morse R, Sanders K, Hows J, et al. (2011). Alkylating chemotherapeutic agents cyclophosphamide and melphalan cause functional injury to human bone marrow-derived mesenchymal stem cells. Ann. Hematol. 90: 777-789.

Kiu H and Nicholson SE (2012). Biology and significance of the JAK/STAT signalling pathways. Growth Factors 30: 88-106.

Kuhn JG (2002). Chemotherapy-associated hematopoietic toxicity. Am. J. Health Syst. Pharm. 59: S4-S7.

Lee SY, Yoon J, Lee MH, Jung SK, et al. (2012). The role of heterodimeric AP-1 protein comprised of JunD and c-Fos proteins in hematopoiesis. J. Biol. Chem. 287: 31342-31348.

Liu C, Li J, Meng FY, Liang SX, et al. (2010). Polysaccharides from the root of Angelica sinensis promotes hematopoiesis and thrombopoiesis through the PI3K/AKT pathway. BMC Complement. Altern. Med. 10: 79.

López-Ruiz P, Rodriguez-Ubreva J, Cariaga AE, Cortes MA, et al. (2011). SHP-1 in cell-cycle regulation. Anticancer Agents Med. Chem. 11: 89-98.

Ma J, Liang QD, Ma ZC, Wang YG, et al. (2010). Rehmanniae Radix provides most of the free fructose and glucose in Si-Wu-Tang decoction. Drug Discov. Ther. 4: 179-183.

Ming M, Wang S, Wu W, Senyuk V, et al. (2012). Activation of Wnt/beta-catenin protein signaling induces mitochondriamediated apoptosis in hematopoietic progenitor cells. J. Biol. Chem. 287: 22683-22690.

Puhalla S, Bhattacharya S and Davidson NE (2012). Hematopoietic growth factors: personalization of risks and benefits. Mol. Oncol. 6: 237-241.

Staal FJ and Luis TC (2010). Wnt signaling in hematopoiesis: crucial factors for self-renewal, proliferation, and cell fate decisions. J. Cell Biochem. 109: 844-849.

Turpaev KT (2006). Role of transcription factor AP-1 in integration of cellular signalling systems. Mol. Biol. (Mosk) 40: 945-961.

Zhang W, Yao H, Wang S, Shi S, et al. (2012). Wnt3a is involved in the early stage of miPSC and mESC haemopoietic differentiation. Cell Biol. Int. 36: 267-271.

Zheng KY, Choi RC, Cheung AW, Guo AJ, et al. (2011). Flavonoids from Radix Astragali induce the expression of erythropoietin in cultured cells: a signaling mediated via the accumulation of hypoxia-inducible factor-1alpha. $J$. Agric. Food Chem. 59: 1697-1704. 